\title{
Model-Based Control of a Large-Scale Ball-on-Plate System With Experimental Validation
}

\author{
Adam Kastner, Jairo Inga, Thomas Blauth, Florian Köpf, Michael Flad, Sören Hohmann \\ Institute of Control Systems (IRS), Karlsruhe Institute of Technology (KIT), Germany \\ adam.kastner@student.kit.edu, jairo.inga@kit.edu
}

\begin{abstract}
A ball-on-plate system is a widespread educationoriented laboratory experiment for automation in mechatronics. The setup combines elements of mechanical, electrical and control engineering and is an adequate setup for learning the combination of theory and practice. This paper presents an example of a workshop result on automatic control in mechatronics. The aim of the workshop is to develop and compare model-based approaches for ball position control in a given large-scale ball-on-plate system. The result includes the derivation of a non-linear state space model of the system. The equations are linearized in the center of the horizontal plate as an operating point in order to apply cascade control, linear-quadratic optimal control and PI optimal state feedback control. The algorithms are implemented on a microcontroller and tested in the experimental setup. The results show a successful control development which achieves the control goal with good performance in terms of command response.
\end{abstract}

\section{INTRODUCTION}

Technological advancements have led to a rising number of mechatronic systems in today's industry but also in daily life. Given the importance of the necessary synergetic work across the fields of mechanical engineering, electrical engineering, automatic control and computer science, it is reasonable that education in engineering should include the development of multidisciplinary skills. In this context, providing laboratory experiments including subsystems from all of these domains plays a crucial role in order to prepare students for practical challenges in engineering.

The ball-on-plate system is an example of a system showing the integration of all of these domains in a clear way. The system consists of a plate, the rotation axes of which can be controlled in order to regulate the position of a ball rolling on it. This system is considered a useful experimental setup for education in mechatronics since it combines mechanics (actuation mechanism), electronics (e.g. sensor and actor setup), as well as computer science (image processing for ball detection) and automation (control of the ball position) in an illustrative way [1]. The development of a real system contributes to learning about practical challenges like the integration of all subsystems and the adequate communication of all components.

We developed a ball-on-plate system to be used as an experimental setup in a workshop focused on automation in mechatronics. The automatic control of a real ball-on-plate system entails several challenges, for example friction effects and non-linearity and open-loop instability of the multipleinput multiple-output system.
The ball-on-plate systems which can be found in literature vary in size, mechanical principles for the plate tilting and the used sensors for measuring plate angles and the ball position. The systems presented in [2] and [3] include a linkage system below the plate. The plate is a touchscreen which determines the ball position. In [4], a different actuation approach with 5 linkages positioned partly around the plate was presented, using a webcam for ball tracking. There is also a commercially available ball-on-plate system [5] which is used in [6]. As for the proposed control strategies, most approaches include a cascade structure [2], [3], [6], [7], where the dynamics of the inner loop are assumed to be fast enough due to the use of servomotors. Therefore, the different applied control methods, e.g. LQ optimal control [3], [7], PD with fuzzy supervisor [4] and sliding mode control [4], [7] are designed only for the outer control loop.

Our ball-on-plate system with a $1 \mathrm{~m}^{2}$ square plate is considerably larger than all systems found in literature (see e.g. the $400 \mathrm{~mm} \times 400 \mathrm{~mm}$ plate in [5]), leading to a greater moment of inertia. For this reason, the aforementioned assumptions concerning the inner loop cannot be made and it is necessary to determine motion equations and controllers for the complete system, including the inner loop and the actuators. A similar modeling approach was done in [8] in order to apply LQ control, but was only tested in simulations.

Thus, the aim of the workshop consists in the design and implementation of a model-based controller in order to balance the ball at any desired position on the plate. Since the mechanical properties of our large-scale ball-on-plate system are different from the state of the art, a mathematical model of the system based on given drawings and 3D-models as well as the datasheets of all components is required. The controller is then designed on this basis. Adequate parameters have to be determined in order to assure adequate control response and disturbance rejection. The next step consists in the implementation of the control algorithm on a microcontroller in order to apply it on the real system. In this way, the performance of the developed control algorithms shall be evaluated.

In this paper, we show a complete modeling and control design process for a new large-scale ball-on-plate system performed by one undergrad student.

The remainder of the paper is organized as follows. Section II presents the main aspects of the given ball-on-plate system. In Section III, a mathematical model of the system 


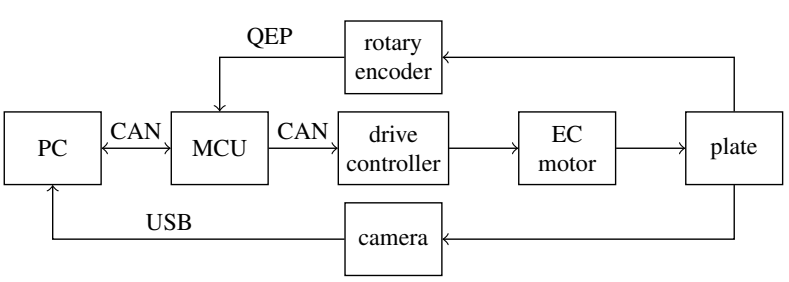

Fig. 1. System architecture

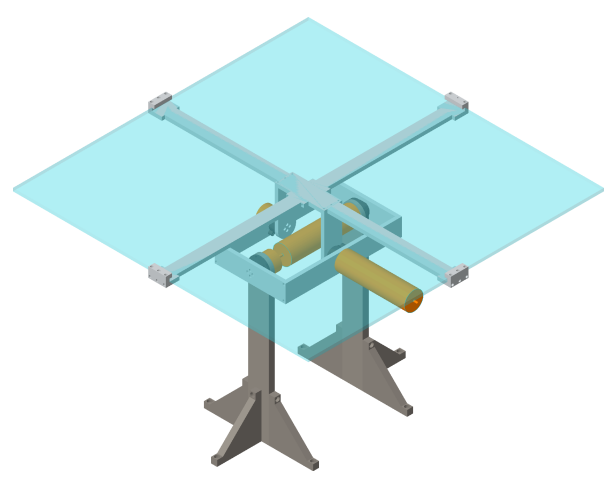

Fig. 2. Ball-on-plate experimental setup

is derived. Section IV is devoted to the chosen controllers to be implemented. The experimental results are presented in Section V. Finally, we draw conclusions in Section VI.

\section{System Description}

This section describes the architecture of the experimental setup, the actuation mechanism used to change the plate inclination and the sensing mechanisms used to measure the plate inclination and ball position.

\section{A. System Architecture}

The system architecture is shown in Fig. 1. Its main components are the following:

- the mechanical assembly with a square acrylic glass plate of size $1 \mathrm{~m}^{2}$ and a frame holding the components

- 2 electronically commutated (EC) geared motors (ebmpapst ECI-63.40-K1-B00 with $i=26$ planetary gearbox)

- 2 motor drive controllers (miControl mcDSA-E40-HC)

- 2 rotary encoders (SICK DFS60A-BHPA65536)

- a camera (IDS UI-3160CP)

- a microcontroller (MCU, Texas Instruments TMS320 F28335)

- a personal computer (PC)

Communication between the microcontroller and the drive controllers is performed via CAN bus using the CANopen protocol. The rotary encoders are connected to the MCU directly using the quadrature encoder pulse (QEP) interface. The PC is connected to the ball-on-plate system using a second CAN bus, where a custom protocol is used.

\section{B. Actuation Mechanism}

Due to the large size of the plate, a design goal of the actuation mechanism was to have no components horizontally beside the plate. Revolute joints with perpendicular

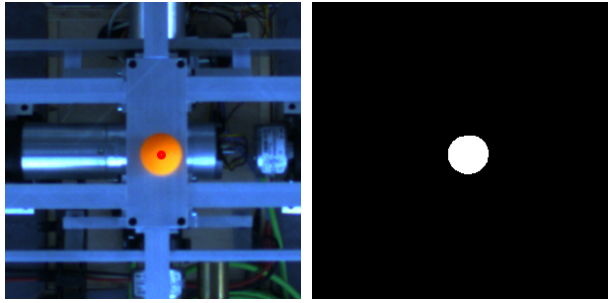

Fig. 3. Original and color threshold filtered camera image

axes in serial configuration, shown in Fig. 2, were placed below the plate to achieve a small footprint. The first joint connects the base frame to an intermediary frame, the second joint connects the intermediary frame to the bracket holding the plate. This mechanism requires a vertical offset between the axes of rotation and the plate. The system acts as an inverse pendulum because the center of mass $(\mathrm{CoM})$ of the plate and bracket assembly is above the axes of the joints. Because the plate surface is above the joint axes, tilting the plate in one direction will cause the ball to move up the slope initially before it is accelerated downwards by gravity, this nonminimum phase behavior must be considered when designing a controller.

\section{Sensing Mechanisms}

Rotary encoders are used to measure the current joint angles $\alpha_{1}$ and $\alpha_{2}$. The plate is in a horizontal position for $\alpha_{1}=\alpha_{2}=0$. Within the camera image the position of the ball is determined using threshold based filtering in the HSV color space, the result of this is shown in Fig. 3. The marked corners of the plate are also detected, allowing the coordinates of the ball $p_{1}$ and $p_{2}$ relative to the plate center to be calculated using a homography. The machine vision algorithm is executed on the PC.

\section{MODELING}

In this workshop run, a new mathematical model is developed to represent the experimental setup because no existing model accounted for both the dynamics of the plate and an offset between plate and joint axes. A nonlinear model is created to simulate system behavior and it is then linearized for controller design. The physical properties of the setup that are used as model parameters are listed in Table I.

TABLE I

MODEL PARAMETERS

\begin{tabular}{|l|l|r|}
\hline Parameter & Symbol & Value \\
\hline radius of ball & $r_{\mathrm{B}}$ & $0.02 \mathrm{~m}$ \\
\hline mass of ball & $m_{\mathrm{B}}$ & $0.0027 \mathrm{~kg}$ \\
\hline mass of plate & $m_{\mathrm{P}}$ & $13.83 \mathrm{~kg}$ \\
\hline moment of inertia of ball & $J_{\mathrm{B}}$ & $7.2 \times 10^{-7} \mathrm{~kg} \mathrm{~m}^{2}$ \\
\hline moment of inertia of plate about axis 1 & $J_{1}$ & $1.545 \mathrm{~kg} \mathrm{~m}^{2}$ \\
\hline moment of inertia of plate about axis 2 & $J_{2}$ & $1.327 \mathrm{~kg} \mathrm{~m}^{2}$ \\
\hline height of ball CoM above axes & $h_{\mathrm{B}}$ & $0.172 \mathrm{~m}$ \\
\hline height of plate CoM above axes & $h_{\mathrm{P}}$ & $0.145 \mathrm{~m}$ \\
\hline effective motor constant & $k_{\mathrm{M}}$ & $0.8508 \mathrm{~N} \mathrm{~m} / \mathrm{A}$ \\
\hline gravity & $g$ & $9.81 \mathrm{~m} / \mathrm{s}^{2}$ \\
\hline
\end{tabular}




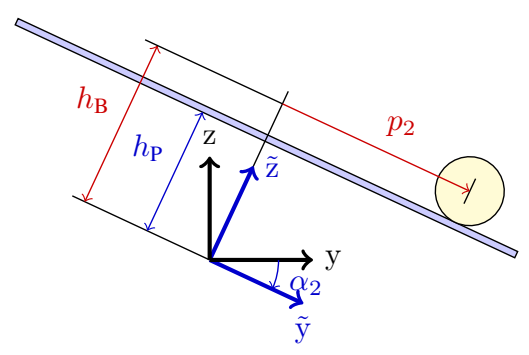

Fig. 4. Coordinates in the $\mathrm{y}-\mathrm{z}$ plane showing a rotation around the $\mathrm{x}$-axis

\section{A. Modeling Procedure}

For modeling the behavior of the ball-on-plate system, the following assumptions are used:

- There is no sliding motion of the ball and it never loses contact with the plate.

- Frictional forces $F_{\mathrm{f}}$ and torques $M_{\mathrm{f}}$ will later be assumed to be zero.

- The change in the moment of inertia of the plate assembly around the first joint axis depending on the rotation of the second joint is neglected.

- The dynamic behavior of the motor drive control loop is neglected, and the joint motor torque is assumed to be $M_{\mathrm{M}}=k_{\mathrm{M}} I$.

Equations of motion for the mechanical system are derived using Lagrange's equation

$$
\begin{aligned}
Q_{i}^{*} & =\frac{\mathrm{d}}{\mathrm{d} t}\left(\frac{\partial L}{\partial \dot{q}_{i}}\right)-\frac{\partial L}{\partial q_{i}} \quad i \in\{1,2,3,4\} \\
L & =E_{\text {kin }}-E_{\text {pot }}
\end{aligned}
$$

where $E_{\text {kin }}$ designates the kinetic and $E_{\text {pot }}$ the potential energy of the system.

The four generalized coordinates are the joint angles $\alpha_{1}=$ $q_{1}$ and $\alpha_{2}=q_{2}$ and the coordinates of the ball $p_{1}=q_{3}$ and $p_{2}=q_{4}$ relative to the center of the plate. The external generalized forces acting on the joints are the sum of friction and motor torques: $Q_{1}^{*}=M_{\mathrm{f}, 1}+M_{\mathrm{M}, 1}, Q_{2}^{*}=M_{\mathrm{f}, 2}+M_{\mathrm{M}, 2}$. No external force except for friction acts on the ball directly: $Q_{3}^{*}=F_{\mathrm{f}, 1}, Q_{4}^{*}=F_{\mathrm{f}, 2}$.

To calculate the energy in the system, the coordinates in a plate-based coordinate system $\{\tilde{x}, \tilde{y}, \tilde{z}\}$ of the centers of mass of the ball

$$
\mathbf{p}_{\mathrm{B}}=\left(\begin{array}{lll}
p_{1} & p_{2} & h_{\mathrm{B}}
\end{array}\right)^{T}
$$

and the plate assembly

$$
\mathbf{p}_{\mathrm{P}}=\left(\begin{array}{lll}
0 & 0 & h_{\mathrm{P}}
\end{array}\right)^{T}
$$

are transformed into a fixed coordinate system $\{\mathrm{x}, \mathrm{y}, \mathrm{z}\}$. The transform, shown in Fig. 4 for $\alpha_{1}=0$, is described by the rotation:

$$
\mathbf{R}=\left(\begin{array}{ccc}
\cos \alpha_{1} & -\sin \alpha_{1} \sin \alpha_{2} & \cos \alpha_{1} \sin \alpha_{2} \\
0 & \cos \alpha_{2} & \sin \alpha_{2} \\
-\sin \alpha_{1} & -\cos \alpha_{1} \sin \alpha_{2} & \cos \alpha_{1} \cos \alpha_{2}
\end{array}\right)
$$

Using this, the potential energy

$$
E_{\mathrm{pot}}=\left(\begin{array}{lll}
0 & 0 & g
\end{array}\right) \mathbf{R}\left(m_{\mathrm{B}} \mathbf{p}_{\mathrm{B}}+m_{\mathrm{P}} \mathbf{p}_{\mathrm{P}}\right)
$$

is calculated from the z-coordinate of the centers of mass in the fixed coordinate system. The kinetic energy

$$
\begin{aligned}
E_{\text {kin }}=\frac{J_{1}}{2} & \dot{\alpha}_{1}^{2}+\frac{J_{2}}{2} \dot{\alpha}_{2}^{2}+\frac{m_{\mathrm{B}}}{2}\left|\frac{\mathrm{d}}{\mathrm{d} t}\left(\mathbf{R p}_{\mathrm{B}}\right)\right|^{2} \\
+ & \frac{J_{\mathrm{B}}}{2}\left(\left(\dot{\alpha}_{1}+\frac{\dot{p}_{1}}{r_{\mathrm{B}}}\right)^{2}+\left(\dot{\alpha}_{2}+\frac{\dot{p}_{2}}{r_{\mathrm{B}}}\right)^{2}\right)
\end{aligned}
$$

is the sum of the rotational energies of plate and ball and the translational energy of the ball. The velocity is calculated as the derivative of the position in the fixed coordinate system.

Using Lagrange's equation yields the nonlinear equations of motion given in Table II. To allow for the use of standard tools and algorithms for control design, a linearization is performed using a steady state with the ball in the center of the horizontal plate as operating point. This leads to a decoupling of the two axes of motion of the ball with similar behavior and differing only in the values of constants.

\section{B. Single Axis Linear State-Space Model}

The linearized equations of motion describe two similar systems for each axis $j \in\{1,2\}$ of motion of the ball, each of which can be represented with a state-space model with four states

$$
\mathbf{x}=\left(\begin{array}{llll}
p_{j} & \dot{p}_{j} & \alpha_{j} & \dot{\alpha}_{j}
\end{array}\right)^{T}
$$

for ball position and velocity as well as plate angle and angular velocity. The system input

$$
u=I_{j}
$$

is the reference current for the motor driver controllers. The standard state-space form

$$
\dot{\mathbf{x}}=\mathbf{A x}+\mathbf{B} u
$$

is used to describe the dynamic behavior of the system with the matrices

$$
\mathbf{A}=\left(\begin{array}{cccc}
0 & 1 & 0 & 0 \\
a_{21} & 0 & a_{23} & 0 \\
0 & 0 & 0 & 1 \\
a_{41} & 0 & a_{43} & 0
\end{array}\right) \quad \text { and } \quad \mathbf{B}=\left(\begin{array}{c}
0 \\
b_{2} \\
0 \\
b_{4}
\end{array}\right)
$$

where:

$$
\begin{aligned}
a_{21} & =-g m_{\mathrm{B}} r_{\mathrm{B}}\left(J_{\mathrm{B}}+h_{\mathrm{B}} m_{\mathrm{B}} r_{\mathrm{B}}\right) / c_{1} \\
a_{23} & =-g r_{\mathrm{B}}\left(-J_{j} m_{\mathrm{B}} r_{\mathrm{B}}+h_{\mathrm{B}} m_{\mathrm{B}} m_{\mathrm{P}} h_{\mathrm{P}} r_{\mathrm{B}}+c_{2}\right) / c_{1} \\
a_{41}= & g m_{\mathrm{B}}\left(m_{\mathrm{B}} r_{\mathrm{B}}^{2}+J_{\mathrm{B}}\right) / c_{1} \\
a_{43}= & g\left(m_{\mathrm{B}} m_{\mathrm{P}} h_{\mathrm{P}} r_{\mathrm{B}}^{2}+c_{2}\right) / c_{1} \\
b_{2}= & -k_{M} r_{\mathrm{B}}\left(J_{\mathrm{B}}+h_{\mathrm{B}} m_{\mathrm{B}} r_{\mathrm{B}}\right) / c_{1} \\
b_{4}= & k_{M}\left(m_{\mathrm{B}} r_{\mathrm{B}}^{2}+J_{\mathrm{B}}\right) / c_{1} \\
c_{1}= & J_{j} J_{\mathrm{B}}+J_{\mathrm{B}} h_{\mathrm{B}}^{2} m_{\mathrm{B}}+J_{j} m_{\mathrm{B}} r_{\mathrm{B}}^{2} \\
& \quad+J_{\mathrm{B}} m_{\mathrm{B}} r_{\mathrm{B}}^{2}-2 J_{\mathrm{B}} h_{\mathrm{B}} m_{\mathrm{B}} r_{\mathrm{B}} \\
c_{2}= & J_{\mathrm{B}} m_{\mathrm{P}} h_{\mathrm{P}}-J_{\mathrm{B}} m_{\mathrm{B}} r_{\mathrm{B}}+J_{\mathrm{B}} h_{\mathrm{B}} m_{\mathrm{B}}
\end{aligned}
$$

The system output

$$
y=\underbrace{\left(\begin{array}{llll}
1 & 0 & 0 & 0
\end{array}\right)}_{\mathbf{C}} \mathbf{x}=p
$$

is the ball position which is also the control variable. 
TABLE II

NONLINEAR EQUATIONS OF MOTION

$$
\begin{aligned}
& M_{\mathrm{M}, 1}=J_{1} \ddot{\alpha}_{1}+J_{\mathrm{B}} \ddot{\alpha}_{1}+\ddot{\alpha}_{1} m_{\mathrm{B}} p_{1}^{2}+\ddot{\alpha}_{1} m_{\mathrm{B}} p_{2}^{2}+\frac{J_{\mathrm{B}} \ddot{p}_{1}}{r_{\mathrm{B}}}+\ddot{\alpha}_{1} h_{\mathrm{B}}{ }^{2} m_{\mathrm{B}} \cos \left(\alpha_{2}\right)^{2}+2 \dot{\alpha}_{1} m_{\mathrm{B}} p_{1} \dot{p}_{1}+2 \dot{\alpha}_{1} m_{\mathrm{B}} p_{2} \dot{p}_{2}-\ddot{\alpha}_{1} m_{\mathrm{B}} p_{2}^{2} \cos \left(\alpha_{2}\right)^{2} \\
& -g m_{\mathrm{B}} p_{1} \cos \left(\alpha_{1}\right)+h_{\mathrm{B}} m_{\mathrm{B}} \ddot{p}_{1} \cos \left(\alpha_{2}\right)+m_{\mathrm{B}} p_{1} \ddot{p}_{2} \sin \left(\alpha_{2}\right)-m_{\mathrm{B}} \ddot{p}_{1} p_{2} \sin \left(\alpha_{2}\right)-g m_{\mathrm{P}} h_{\mathrm{P}} \cos \left(\alpha_{2}\right) \sin \left(\alpha_{1}\right) \\
& +\dot{\alpha}_{2}^{2} h_{\mathrm{B}} m_{\mathrm{B}} p_{1} \cos \left(\alpha_{2}\right)-g h_{\mathrm{B}} m_{\mathrm{B}} \cos \left(\alpha_{2}\right) \sin \left(\alpha_{1}\right)-\ddot{\alpha}_{1} h_{\mathrm{B}} m_{\mathrm{B}} p_{2} \sin \left(2 \alpha_{2}\right)-\dot{\alpha}_{1} h_{\mathrm{B}} m_{\mathrm{B}} \dot{p}_{2} \sin \left(2 \alpha_{2}\right)-2 \dot{\alpha}_{1} m_{\mathrm{B}} p_{2} \dot{p}_{2} \cos \left(\alpha_{2}\right)^{2} \\
& -\dot{\alpha}_{2}^{2} m_{\mathrm{B}} p_{1} p_{2} \sin \left(\alpha_{2}\right)+g m_{\mathrm{B}} p_{2} \sin \left(\alpha_{1}\right) \sin \left(\alpha_{2}\right)+2 \dot{\alpha}_{1} \dot{\alpha}_{2} h_{\mathrm{B}} m_{\mathrm{B}} p_{2}-\dot{\alpha}_{1} \dot{\alpha}_{2} h_{\mathrm{B}}^{2} m_{\mathrm{B}} \sin \left(2 \alpha_{2}\right)+\dot{\alpha}_{1} \dot{\alpha}_{2} m_{\mathrm{B}} p_{2}^{2} \sin \left(2 \alpha_{2}\right) \\
& +\ddot{\alpha}_{2} h_{\mathrm{B}} m_{\mathrm{B}} p_{1} \sin \left(\alpha_{2}\right)+\ddot{\alpha}_{2} m_{\mathrm{B}} p_{1} p_{2} \cos \left(\alpha_{2}\right)+2 \dot{\alpha}_{2} m_{\mathrm{B}} p_{1} \dot{p}_{2} \cos \left(\alpha_{2}\right)-4 \dot{\alpha}_{1} \dot{\alpha}_{2} h_{\mathrm{B}} m_{\mathrm{B}} p_{2} \cos \left(\alpha_{2}\right)^{2} \\
& M_{\mathrm{M}, 2}=J_{2} \ddot{\alpha}_{2}+J_{\mathrm{B}} \ddot{\alpha}_{2}+\ddot{\alpha}_{2} m_{\mathrm{B}} p_{2}^{2}+h_{\mathrm{B}} m_{\mathrm{B}} \ddot{p}_{2}+\frac{J_{\mathrm{B}} \ddot{p}_{2}}{r_{\mathrm{B}}}+\ddot{\alpha}_{2} h_{\mathrm{B}}^{2} m_{\mathrm{B}}+2 \dot{\alpha}_{2} m_{\mathrm{B}} p_{2} \dot{p}_{2}-\dot{\alpha}_{1}^{2} h_{\mathrm{B}} m_{\mathrm{B}} p_{2}+\frac{\dot{\alpha}_{1}^{2} h_{\mathrm{B}}{ }^{2} m_{\mathrm{B}} \sin \left(2 \alpha_{2}\right)}{2} \\
& -\frac{\dot{\alpha}_{1}^{2} m_{\mathrm{B}} p_{2}^{2} \sin \left(2 \alpha_{2}\right)}{2}-g m_{\mathrm{P}} h_{\mathrm{P}} \cos \left(\alpha_{1}\right) \sin \left(\alpha_{2}\right)-g h_{\mathrm{B}} m_{\mathrm{B}} \cos \left(\alpha_{1}\right) \sin \left(\alpha_{2}\right)-g m_{\mathrm{B}} p_{2} \cos \left(\alpha_{1}\right) \cos \left(\alpha_{2}\right) \\
& +2 \dot{\alpha}_{1}^{2} h_{\mathrm{B}} m_{\mathrm{B}} p_{2} \cos \left(\alpha_{2}\right)^{2}+\ddot{\alpha}_{1} h_{\mathrm{B}} m_{\mathrm{B}} p_{1} \sin \left(\alpha_{2}\right)+2 \dot{\alpha}_{1} h_{\mathrm{B}} m_{\mathrm{B}} \dot{p}_{1} \sin \left(\alpha_{2}\right)+\ddot{\alpha}_{1} m_{\mathrm{B}} p_{1} p_{2} \cos \left(\alpha_{2}\right)+2 \dot{\alpha}_{1} m_{\mathrm{B}} \dot{p}_{1} p_{2} \cos \left(\alpha_{2}\right) \\
& 0=\dot{\alpha}_{1}^{2} m_{\mathrm{B}} p_{1}-m_{\mathrm{B}} \ddot{p}_{1}+g m_{\mathrm{B}} \sin \left(\alpha_{1}\right)-\frac{J_{\mathrm{B}} \ddot{\alpha}_{1}}{r_{\mathrm{B}}}-\frac{J_{\mathrm{B}} \ddot{p}_{1}}{r_{\mathrm{B}}{ }^{2}}-\ddot{\alpha}_{1} h_{\mathrm{B}} m_{\mathrm{B}} \cos \left(\alpha_{2}\right)+\ddot{\alpha}_{1} m_{\mathrm{B}} p_{2} \sin \left(\alpha_{2}\right)+2 \dot{\alpha}_{1} m_{\mathrm{B}} \dot{p}_{2} \sin \left(\alpha_{2}\right) \\
& +2 \dot{\alpha}_{1} \dot{\alpha}_{2} h_{\mathrm{B}} m_{\mathrm{B}} \sin \left(\alpha_{2}\right)+2 \dot{\alpha}_{1} \dot{\alpha}_{2} m_{\mathrm{B}} p_{2} \cos \left(\alpha_{2}\right) \\
& 0=\dot{\alpha}_{1}^{2} m_{\mathrm{B}} p_{2}-m_{\mathrm{B}} \ddot{p}_{2}+\dot{\alpha}_{2}^{2} m_{\mathrm{B}} p_{2}-\ddot{\alpha}_{2} h_{\mathrm{B}} m_{\mathrm{B}}-\frac{J_{\mathrm{B}} \ddot{\alpha}_{2}}{r_{\mathrm{B}}}-\frac{J_{\mathrm{B}} \ddot{p}_{2}}{r_{\mathrm{B}}^{2}}-\frac{\dot{\alpha}_{1}^{2} h_{\mathrm{B}} m_{\mathrm{B}} \sin \left(2 \alpha_{2}\right)}{2}-\dot{\alpha}_{1}^{2} m_{\mathrm{B}} p_{2} \cos \left(\alpha_{2}\right)^{2}-\ddot{\alpha}_{1} m_{\mathrm{B}} p_{1} \sin \left(\alpha_{2}\right) \\
& -2 \dot{\alpha}_{1} m_{\mathrm{B}} \dot{p}_{1} \sin \left(\alpha_{2}\right)+g m_{\mathrm{B}} \cos \left(\alpha_{1}\right) \sin \left(\alpha_{2}\right)
\end{aligned}
$$

\section{Control Design}

Multiple controllers are designed based on the linearized model. The control goal of balancing the ball is defined as bringing the ball to a standstill at an arbitrarily defined position on the plate. Two separate controllers with similar structure are used for the two axes of motion of the ball. Continuous-time design procedures were used and the controller is discretized later for implementation.

Three possible control structures are considered and will be compared:

- Cascade control similar to [2] and [6], where the inner loop controls the plate inclination and the outer loop controls the ball position.

- State feedback similar to [8], where the control signal is calculated from the system state $\mathbf{x}$.

- PI state control, where state feedback is combined with a PI controller.

\section{A. Cascade Control}

The cascade control structure shown in Fig. 5 uses an inner loop to control the inclination of the plate $\alpha$ and an outer loop to control the position of the ball $p$. Cascade control is often used with a fast-acting inner loop whose dynamic behavior is not considered when designing the outer loop controller. In this large-scale experimental setup, the high moment of inertia of the plate limits the angular acceleration and thus the response speed of the inner loop, requiring outer controller design to consider the dynamics of the inner loop.

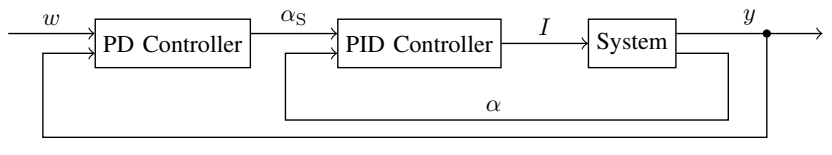

Fig. 5. Cascade Control structure.
The inner loop is designed based on the transfer function between motor current and plate angle, neglecting all influence from the ball:

$$
G_{\mathrm{P}}(s)=\frac{\frac{k_{\mathrm{M}}}{J_{j}}}{s^{2}-\frac{g h_{\mathrm{P}} m_{\mathrm{P}}}{J_{j}}}
$$

To control this unstable second-order system, a PID controller is used. Integral action in the inner loop is used to achieve stationary accuracy despite the gravitational force of the ball acting as a static disturbance.

To avoid large system inputs when a sudden change in command signal $\alpha_{\mathrm{S}}$ occurs, a controller with two degrees of freedom for command and control signal is used and derivative action is only applied to the control signal. The back-calculation algorithm as described in [9] is used for anti-windup.

The outer controller is designed based on the combined transfer function

$$
G_{\mathrm{O}}(s)=G_{\mathrm{I}}(s) \cdot \frac{-r_{\mathrm{B}}\left(J_{\mathrm{B}}+h_{\mathrm{B}} m_{\mathrm{B}} r_{\mathrm{B}}\right) s^{2}+g m_{\mathrm{B}} r_{\mathrm{B}}^{2}}{\left(m_{\mathrm{B}} r_{\mathrm{B}}^{2}+J_{\mathrm{B}}\right) s^{2}}
$$

of the inner loop and the transfer function between plate inclination and ball position. To control the resulting system with double integral behavior, a PD controller is used. The outer controller is also designed as a two-degree-offreedom controller that does not apply derivative action to the command signal.

Appropriate controller gains are determined using the pidTuner tool of the software Matlab.

\section{B. State Feedback}

The state feedback control structure shown in Fig. 6 uses the matrix $\mathbf{K}_{\mathrm{R}}$ to calculate the control signal from the system state $\mathbf{x}$ and achieves stationary accuracy by multiplying the command signal $w$ with the prefactor $k_{\mathrm{V}}$. 


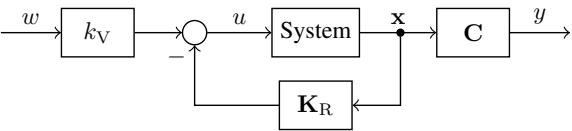

Fig. 6. State Feedback structure.

An optimal state feedback matrix $\mathbf{K}_{\mathrm{R}}$ is calculated as a linear-quadratic regulator $(\mathrm{LQR})$ that minimizes the cost function

$$
J=\int_{0}^{\infty} \mathbf{x}^{T} \mathbf{Q} \mathbf{x}+R u^{2} \mathrm{~d} t
$$

where the states are penalized with the weighting matrix $\mathbf{Q}$ and the control effort with the weight $R$.

To achieve the control goal, the position error must be minimized, which is why the weighting matrix

$$
\mathbf{Q}=\operatorname{diag}\left(Q_{p}, 0,0,0\right)
$$

is chosen to penalize only the position of the ball, where $\operatorname{diag}(\ldots)$ denotes a square diagonal matrix. The prefactor applied to the command signal $w$ must be set to

$$
k_{\mathrm{V}}=-\left(\mathbf{C}\left(\mathbf{A}-\mathbf{B} \mathbf{K}_{\mathrm{R}}\right)^{-1} \mathbf{B}\right)^{-1}
$$

to achieve stationary accuracy as described in [10].

Simulations are used to determine values for $Q_{p}$ and $R$ where the LQR controller achieves fast reaction to set-point changes while avoiding motor current saturation.

\section{PI State Control}

The PI state controller structure shown in Fig. 7 consists of a constant state feedback $\mathbf{K}_{\mathrm{R}}^{*}$ and a PI controller that uses integral action to achieve stationary accuracy even when static disturbances are present.

To calculate appropriate state feedback and controller gains, an extended state space model with state vector

$$
\mathbf{x}_{\mathrm{I}}=\left(\begin{array}{ll}
\mathbf{x}^{T} & z
\end{array}\right)^{T}
$$

is used. It introduces a new state $z$ that represents the integral of the control error $e=w-y$ between set point $w$ and system output $y$ :

$$
\left(\begin{array}{c}
\dot{\mathbf{x}} \\
\dot{z}
\end{array}\right)=\left(\begin{array}{cc}
\mathbf{A} & \mathbf{0} \\
-\mathbf{C} & 0
\end{array}\right)\left(\begin{array}{l}
\mathbf{x} \\
z
\end{array}\right)+\left(\begin{array}{c}
\mathbf{B} \\
0
\end{array}\right) u+\left(\begin{array}{l}
\mathbf{0} \\
1
\end{array}\right) w
$$

An LQR feedback matrix $\mathbf{K}$ is calculated to minimize the cost function:

$$
J=\int_{0}^{\infty} \mathbf{x}_{\mathrm{I}}^{T} \mathbf{Q}_{\mathrm{I}} \mathbf{x}_{\mathrm{I}}+R_{\mathrm{I}} u^{2} \mathrm{~d} t
$$

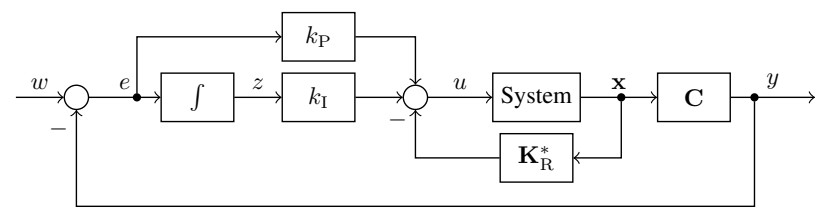

Fig. 7. PI State Controller structure.
To achieve stationary accuracy, the integral $z$ of the control error must be penalized. Because achieving no change in the integral of the control error requires the control error and all other states to be zero, the weighting matrix

$$
\mathbf{Q}_{\mathrm{I}}=\operatorname{diag}\left(0,0,0,0, Q_{z}\right)
$$

is chosen to penalize only $z$.

The resulting state feedback matrix $\mathbf{K}$ for the extended model can be split into the controller gains $k_{\mathrm{I}}$ and $k_{\mathrm{P}}$ and the state feedback matrix $\mathbf{K}_{\mathrm{R}}^{*}$ :

$$
\mathbf{K}=\left(\begin{array}{c}
\mathbf{K}_{\mathrm{R}}^{*}+k_{\mathrm{P}} \mathbf{C} \\
k_{\mathrm{I}}
\end{array}\right)
$$

Simulations show that choosing $k_{\mathrm{P}}>0$ results in large overshoot, which is why $k_{\mathrm{P}}=0$ was used in the final controller. The values for the weights $Q_{z}$ and $R_{\mathrm{I}}$ were chosen based on simulations and tests of the system. Anti-windup is added to the controller using the back-calculation algorithm.

In practice, stiction in the plate joints prevents small changes in plate inclination. Because of this, it is not possible to achieve zero control error between set-point and system output. To prevent the integral action of the controller from setting the system in motion again when the ball is already at a standstill close to the set-point, a dead zone is added to the integral action so that position errors smaller than $\pm 20 \mathrm{~mm}$ are ignored. This prevents an oscillating motion around the set-point.

\section{EXPERIMENTAL RESULTS}

The controllers are implemented on a microcontroller and used to control the experimental setup. Tests are performed for validation and to compare the different structures.

\section{A. Implementation}

The control algorithm is implemented on the MCU using Simulink Coder, a part of the Matlab software package. A time-discretization of the controllers developed in Section IV is performed using a sampling time of $T_{\mathrm{A}}=0.04 \mathrm{~s}$. For approximating the integral and derivative action in the controller, the forward Euler algorithm is used.

State feedback requires all states of the system to be known. In the experimental setup, only the coordinates of the ball $p$ and the joint angles $\alpha$ can be measured directly. The velocity of the ball $\dot{p}$ and the angular velocity $\dot{\alpha}$ of the plate are estimated using the difference quotient.

\section{B. Experimental Validation}

The implemented controllers were tested using the experimental setup. All control structures stabilize the system and they rebalance the ball after a disturbance is introduced by manually tilting the plate or pushing the ball.

Reaction to a set-point change was tested and Fig. 8 shows the response of the experimental setup with all three controller structures to a change in set-point from $-0.15 \mathrm{~m}$ to $+0.3 \mathrm{~m}$ in one axis of motion. The ball is moved close to the new target position and brought to a standstill by all three controllers. A small stationary error remains due 

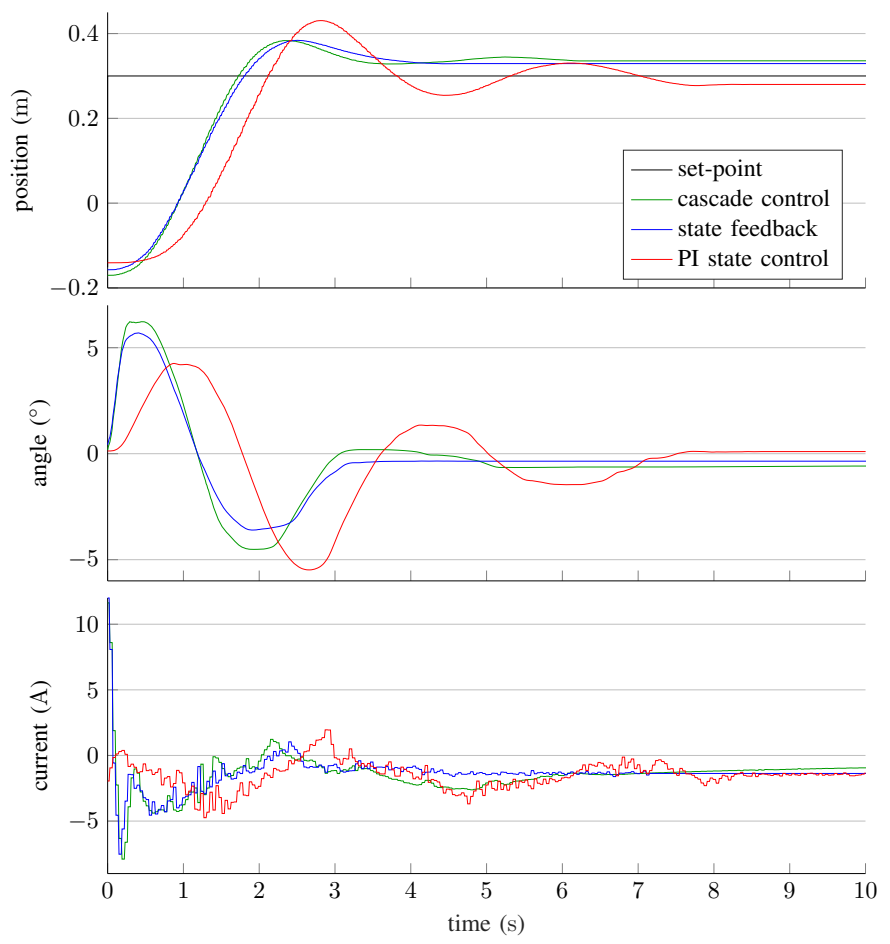

Fig. 8. Step responses of the experimental setup.

to stiction restricting small changes in plate inclination. Stiction between ball and plate causes the ball to remain in a stationary position when plate inclination is small, as can be seen in the different final angle values in Fig. 8 .

Both cascade control and state feedback have a similar short rise time in ball position, with the PI state controller being significantly slower. The settling time needed to bring the ball to a standstill is shortest for state feedback. PI state control causes a short-lived oscillation of the ball around the target position, increasing settling time.

Small fluctuations in the motor current are caused by measurement noise but do not affect control quality. Due to an assymetrical distribution of mass of the plate assembly, a small current is needed to maintain a horizontal plate position and an offset was added to the state feedback for compensation.

\section{Comparison of Control Structures}

The controller structures are compared based on their step response shown in Fig. 8.

A short rise time in ball position is achieved using proportional feed-through of the command signal in cascade control and state feedback, a step in set-point immediately leads to a step in motor current causing a quick change in plate inclination. PI state control achieves set-point following using only integral action, leading to a slower initial rise. Depending on circumstances, it can be preferable to have a motor current that changes smoothly and does not reach high absolute values, reducing strain on the mechanical components.

The PI state controller has the largest settling time, due to its integral action and the delay in the system caused by stiction creating an oscillation. A short settling time can be achieved by using state feedback where the controller has no internal dynamic behavior.

The outer loop of the cascade controller and state feedback do not have integral action, thus losing stationary accuracy when stiction acts as a static disturbance. PI state control guarantees that the stationary control error is within the width of the dead zone, making its performance more reliable than that of the other structures.

\section{CONCLUSION}

We presented in this paper the development of a modelbased controller for a large-scale ball-on-plate system as an example result of a workshop concept on automation in mechatronics. Student feedback included positive comments on the experience of algorithm implementation which allowed learning how to deal with practical challenges, e.g. friction effects. Based on the developed mathematical model, it was possible to design several controllers which achieve the control goal and at the same time discuss their strengths and weaknesses in a real application. This workshop run was performed primarily by one student. In future, we plan to build joint working groups of 3-4 undergrad students which participate on the workshop while attending lectures on practical aspects of control engineering.

\section{REFERENCES}

[1] K. Craig, "Is anything really new in mechatronics education?" IEEE Robotics Automation Magazine, vol. 8, no. 2, pp. 12-19, Jun. 2001.

[2] S. Awtar, C. Bernard, N. Boklund, A. Master, D. Ueda, and K. Craig, "Mechatronic design of a ball-on-plate balancing system," Mechatronics, vol. 12, no. 2, pp. 217-228, 2002.

[3] F. C. Braescu, L. Ferariu, R. Gilca, and V. Bordianu, "Ball on plate balancing system for multi-discipline educational purposes," in 2012 16th International Conference on System Theory, Control and Computing (ICSTCC), Oct. 2012, pp. 1-6.

[4] M. Moarref, M. Saadat, and G. Vossoughi, "Mechatronic design and position control of a novel ball and plate system," in 2008 16th Mediterranean Conference on Control and Automation. IEEE, Jun. 2008, pp. 1071-1076.

[5] "CE 151 Ball \& Plate Model | Humusoft." [Online]. Available: http://www.humusoft.cz/models/ce151/

[6] A. Knuplez, A. Chowdhury, and R. Svecko, "Modeling and control design for the ball and plate system," in IEEE International Conference on Industrial Technology, 2003, vol. 2, Dec. 2003, pp. 1064-1067 Vol.2.

[7] A. Kassem, H. Haddad, and C. Albitar, "Commparison Between Different Methods of Control of Ball and Plate System with 6dof Stewart Platform," IFAC-PapersOnLine, vol. 48, no. 11, pp. 47-52, 2015.

[8] F. Dušek, D. Honc, and K. R. Sharma, "Modelling of ball and plate system based on first principle model and optimal control," in 2017 21st International Conference on Process Control (PC), Jun. 2017, pp. 216-221.

[9] K. J. Åström and T. Hägglund, Advanced PID control. Research Triangle Park, NC: ISA - Instrumentation, Systems, and Automation Society, 2006.

[10] R. L. Williams, D. A. Lawrence et al., Linear state-space control systems. John Wiley \& Sons, 2007. 